\title{
Clarifying Incoherence in Games
}

\author{
Hugo Dirk Hogenbirk \\ Marries van de Hoef \\ John-Jules Charles Meyer
}

In this paper we will analyze the concept of incoherency that has been put forward by Jesper Juul in Half-Real (2005). Juul provides a paradigmatic example of incoherence in the game Donkey Kong (Nintendo 1981). The main character of the narrative, Mario, can die and subsequently reappear at the beginning of the level. However, when pressed to describe the narrative of the game, most players would not say that Mario ever died. The respawn is attributed to the game rules instead. Juul holds that this phenomenon constitutes an incoherence in the game's fictional world. We propose to call this form of incoherence for form-fiction incoherence and claim that Juul is unclear about the precise nature of this phenomenon. We argue that Juul's attempt to elucidate this form of incoherence as a form of contradiction is mistaken. Furthermore, we argue that Edward Wesp, in his discussion of Juul's use of the notion of incoherence incorrectly identifies the concept with "incompleteness" (Wesp 2014). We propose to redefine the incoherence phenomenon as a "pseudo fictional event." Subsequently, we provide an explanation for what underlies an incoherence by adopting a variant of the principle of charity (Davidson 1973). Lastly we discuss how a proper understanding of incoherence can help game designers and how it relates to ludonarrative dissonance (Hocking 2009).

Keywords: fictional worlds, incoherency, rule-fiction interaction

\section{Introduction}

Jesper Juul describes games in Half Real (2005) as consisting of two parts: a formal rule system and a fictional world. Juul here makes use of the concept of a fictional world introduced by Thomas Pavel (1986), who applies possible world semantics to fiction. Juul identifies an interesting property of the fictional worlds of games: they can be "incoherent" in relation to the rules of the game. This form of incoherence is identified by Juul as a property of the fictional worlds of games. (Juul 2005, 123, 130, 207). Incoherence is introduced as the phenomenon that while certain events happen on our screens, we do not take them to have actually happened; they are not taken to be a part of the fictional world. However, criticisms have been raised at this idea of incoherence and its supposedly privileged relation to video games by Edward Wesp in "A Too-Coherent World: Game Studies and the Myth of 'Narrative' Media” (2014). The views of both these authors will be discussed in this paper. 
The word "incoherence" can be used in a number of different ways. Juul himself is not consistent in his use of the term, and appears to be using different concepts at different times. Wesp interprets Juul's use of incoherence as a form of incompleteness of a fictional world. We will look at whether these different interpretations apply to the Mario example. We will conclude that there is severe confusion in the use of the concept of incoherence that Juul has introduced. Clearing up this confusion, and finding the core of the phenomenon that Juul has drawn attention to, will give us a clarified and powerful concept of incoherence.

Juul's concept of incoherence is tied to the way the rules and fiction of a game can interact and collide. We can understand this as the form of the medium (in this case the rules) interacting with the fiction (or content) expressed by the medium. For this reason, and conceptual clarity, we will refer to the phenomenon Juul has drawn attention to as form-fiction incoherence. Implications for game design will be discussed in the final section of this paper.

\section{The Phenomenon}

In Juul's book Half-Real we find his paradigmatic example of an incoherence (which we have dubbed a form-fiction incoherence). We will refer to this as "the Mario example”. Juul writes:

( ... ) it is possible to imagine a world in which Mario's girlfriend is kidnapped by an evil gorilla and has to be rescued. ( ... ) It is harder to understand why Mario has three lives: Being hit by a barrel, by a fireball, or by an anvil should reasonably be fatal. Furthermore, the player is rewarded with an extra Mario at 10.000 points. (Juul 2005, 123)

In the arcade game Donkey Kong we follow the protagonist Mario. The narrative of the story is that Mario's girlfriend has been kidnapped by the aforementioned evil gorilla, Donkey Kong. We play as Mario and need to work our way up to the gorilla to save our girlfriend, in the meantime overcoming perilous obstacles.

However, when taking these events and characters as the whole story, or better, as a good description of what happens in the game's fictional world, there are events that seem to appear on our screens that do not make any sense. The main example Juul uses is Mario's seeming ability to reincarnate. When we play the game, and something happens to Mario that should kill him (being hit by the barrel etc.) he disappears for a second, a number on the top right side of the screen decreases by one, and Mario reappears in the bottom left side of the screen. What conclusions regarding the fictional world of Donkey Kong should we draw from this information?

Of course, when playing the game, we are not troubled by this issue for long. We are aware that the events we just observed should not be incorporated into the fiction of the game. 
Mario did not die and reincarnate; we as players just got a second chance at playing the game. Or as Juul puts it:

Mario is not reincarnated (fiction); the player just has three Mario's (rules). (Juul 2005, 130)

We do not add information about the events displayed on our screens to the fictional world. However, this fact alone does not necessarily raise a noteworthy point about the fiction of the game. There are examples where we can explain, without recourse to rules, that we should not understand some information as being part of the fictional world. For example, we are also presented with proof of the world being plastered over by the word "PAUSE" when we press the pause-button. Again, we do not take this evidence to indicate the existence of the word "PAUSE" in the fictional world of Donkey Kong. In the case of the pause-button, we seem to be presented with an "overlay" of the game. None of the fictional objects we already have accepted to be in the fictional world are tied to this word. In the Mario example, however, a like argument cannot be made. Here, arguably the most important entity of the fictional world, the protagonist Mario, disappears and reappears when being inflicted with lethal wounds. We cannot claim that this event should "clearly" be disregarded. More explanation is necessary: why do we disregard this event and not other events happening to Mario? One of Juul's descriptions of what happens in the Mario example will help us here:

( ... ) we call this type of fictional world incoherent, meaning that there are many events in the fictional world that we cannot explain without discussing the game rules (Juul 2005, p.130)

[emphasis ours]

What Juul points to here is that the events that happen on our screens need to be explained away and they are explained away by reference to the game's rules. So in the Mario example the form-fiction incoherence occurs because something that happens on our screen is not taken to be a part of the fiction of the game; it is explained away by means of the game's rules.

\section{Fictional Worlds and Game Environments}

Something puzzling about the Mario example we have just discussed is the following: on the one hand we understand that Mario does not reincarnate, but we would only claim this aloud because we see there is some sense in which Mario does reincarnate. We could also state that Mario cannot fly, but this is uninteresting precisely because we see no reason to assume that he can fly. We see Mario dis- and re- appearing on the screen (in this sense the reincarnation-event occurs) but we decide against accepting that it actually happened. To account for this oddity, let us fix some terminology.

The fictional world of Donkey Kong is comprised of exactly all those propositions regarding the fiction of Donkey Kong we would assert as being true (Pavel 1986, 50). Examples of these propositions are “Donkey Kong has captured Mario’s girlfriend”, 
"Mario is trying to save his girlfriend", "Donkey Kong throws barrels to keep Mario from saving his girlfriend" etc. One of the main reasons for Juul to assert that incoherence has occurred is that when I retell the story of what happened while I played Donkey Kong I do not claim Mario to reincarnate (Juul 2005, 124). The story we tell of what happened in the game reflects on what we do and we do not assert to be the case of the fiction and the fictional world. So, the sense in which Mario did not reincarnate is that in the fictional world, Mario did not reincarnate.

But in what sense did Mario reincarnate? Mario seemed to reincarnate because we were presented with his dis- and re-appearance in the gaming environment. The game presented us with audio-visual feedback that, at face value, gave us reason to assert that Mario disappears and reappears. From this event, we might further conclude that Mario for example reincarnated. So we can then answer "in what sense did Mario reincarnate?" by stating that in the game environment, Mario did reincarnate.

The difference between events in the fictional world and events in the game environment will make easily expressible that while we have some reason (an event in the game environment) to claim Mario reincarnated, we claim that in the fictional world he did not do so. ${ }^{1}$

In the next sections we will examine both Juul's and Wesp's claims and show that these interpretations do not accurately describe the Mario example and thus should not be taken as acceptable ways to understand the concept of form-fiction incoherence. In the next two sections we will discuss what form-fiction incoherence is not. After this discussion we will outline our own notion of what it is.

\section{What Form-fiction Incoherence is Not: Contradictions}

One of the ways Juul proposes to understand incoherence is by identification of form-fiction incoherence of the fictional world with the occurrence of contradictions in the game environment, as we can see from the following remark:

( ... ) some games, and many video games, present game worlds that are incoherent worlds, where the game contradicts itself or prevents the player from imagining a complete fictional world. (Juul 2005, 123)

It is the first part of the disjunction that we will talk about here, the occurrence of contradictions in the game environment (in Juul here more or less denoted by the term

1 We speak here of reincarnation and in this we follow Juul's example. However, where there to be some other natural interpretation to Mario's dis- and re-appearance readily available that would not be troubling. What is important is the strange way we find ourselves disregarding the information presented to us by the game, and not what exactly the interpretation of this information would have been were it not have been disregarded. 
game world). We will argue that identifying form-fiction incoherence with contradictions is not a sensible explanation of this phenomenon.

Firstly, contradictions are not a necessary condition for the occurrence of formfiction incoherence. Take the Mario example. There is no contradiction in neither game environment nor fictional world involved in this example. A contradiction would be when both $A$ and not $A$ would be the case. But, there is nothing distinctly contradictory about what we are presented with in the game environment; someone disappearing and reappearing is not contradictory, despite being potentially weird. No contradiction is presented to us in the game environment.

Nor does there appear to be a contradiction in the fictional world, neither if we take Mario to have reincarnated nor when we take Mario to not have reincarnated. If we do not take what we see occur in the game environment seriously, and disregard the events we observe on our screens, Mario did not reincarnate in the fictional world (which seems to be the preferable interpretation, but not because we would contradict ourselves otherwise). If we do take what we see seriously, Mario did reincarnate in the fictional world (which might be an unpreferable interpretation, but again, not on pains of being contradictory with anything else in the fictional world). We can give an interpretation of what has happened; if the fictional world were truly contradictory, this would not have been possible.

One might be tempted to claim that a "contradiction" occurs between the game environment and the fictional world if we accept that we should not take seriously the disand re- appearance of Mario in the game environment. But this is not the case precisely because we understand these two 'places' to be distinct. ${ }^{2}$ If we were obliged to take everything at face value, as we do in the game environment, then holding Mario not to reincarnate would lead to contradiction, for then Mario did and did not reincarnate. But, we understand that what the screen shows us at face value does not have to be true of the fictional world. What however is the case is that the game environment and the fictional world differ exactly in this: whether Mario dis- and re- appeared. The Mario example does not contain contradictions, so contradictions cannot be a necessary condition for form-fiction incoherence to occur.

Contradictions are not sufficient conditions for form-fiction incoherence either. Let us posit that in the game environment (i.e. at face value), a contradiction does occur. For example say that the game starts with the following message "Mario is wearing a black cap” while on screen he is clearly wearing a red one. Are we now required to conclude that this is a case of form-fiction incoherence? We are not because, firstly, there will be

2 Or as Pavel puts it, of some fictional world I do not have to accept that it overlaps completely with the presentation of that fictional world (Pavel 1986, 50). 
no recourse to the rules to explain this event. Secondly, we will most likely not simply keep this information out of the fictional world, like we did with Mario's dis- and reappearing. When we would be pressed about whether Mario reincarnated, we would answer with a resounding no. However, when asked about the color of the cap, we would probably respond that we're not sure, or that there is no determinate color of the cap, or perhaps, that it is either red or black. This shows that contradictions in the game environment are neither a necessary nor a sufficient condition for form-fiction incoherence. Contradictoriness cannot be a sensible interpretation of form-fiction incoherence.

However, that is not to say contradictions and form-fiction incoherence have to be unrelated to each other. We claim that although the occurrence of a contradiction in the game environment cannot be an occurrence of form-fiction incoherence, the contradiction may lead to it. Let us give an example. Many games present us with game environments in which the following can happen: some protagonist will tell us we will never be able to take on hundreds of enemies on our own. And this claim seems to us completely sensible (the protagonist is just a regular soldier). However, as players, we have put the game on "very hard mode", so to succeed we are now faced with hundreds of enemies (where normally the level would consist of twenty enemies). And lo and behold, the same character who just told us we cannot take on hundreds of enemies is doing exactly that, or so our screens are showing us. And here we have a contradiction in the game environment leading to form-fiction incoherence; in the fictional world the protagonist could indeed not take on hundreds of enemies and the killing of hundreds of enemies needs to be explained and subsequently disregard by recourse to the rules. ${ }^{3}$

So contradictions in the game environment may spark form-fiction incoherence, as our example shows. But as we argued before, we should not confuse this with the identification of form-fiction incoherence and contradictions.

\section{What Form-fiction Incoherence is Not: Incompleteness}

In the article "A Too-Coherent World: Game Studies and the Myth of "Narrative" Media” by Edward Wesp (2014), it is claimed that the phenomenon Juul is referring to is not a matter of incoherence but is a form of incompleteness. In this section we will argue against this claim. But before we do this, we need to shortly examine what incompleteness is in the context of fictional worlds. In his book "Fictional Worlds" Thomas Pavel (1986) makes a theory of fictional worlds, in analogy to the theory of possible worlds, which is also applied by Juul and by extension Wesp.

3 The gameplay element we would we invoke here would then be the difficulty setting. 
One important aspect of this theory is that fictional worlds can be incomplete. Pavel puts it like this:

About complete worlds, one can decide whether for any proposition $p$, either $p$ or its negation non- $p$ is true in that world. But how can we decide whether "Vautrin has a cousin" and "Lady Macbeth has four children" are true or false in their respective fictional worlds? (Pavel 1986, p.107) ${ }^{4}$

So, fictional worlds can be so that we cannot decide that some proposition is true or false. We can ask about the fictional world of Donkey Kong whether, a week after saving his girlfriend, Mario will eat an apple for breakfast. However, about this we should claim that this is simply not decided for the fictional world. Wesp's claim would then be that occurrences of what we have called form-fiction incoherence are not in any principled way different from occurrences of incompleteness.

To argue this, Wesp writes that Juul makes no clear distinction between what we have dubbed form-fiction incoherence and incompleteness in his definition of incoherence, a problem he holds is aggravated by the Mario example:

So while Donkey Kong does prevent the player from gathering a specific, diegetic understanding of Mario's ability to resume his rescue attempt after his apparent demise, it is not clear why that gap creates a uniquely "incoherent" situation relative to the incompletions that Juul himself sees as part and parcel of fiction overall. (Wesp 2014)

Wesp claims there is nothing uniquely incoherent in the Mario example compared to other media. The reason Wesp thinks this is the case is because incoherence, when understood as the inability to gather a complete understanding of the fictional world, is a form of incompleteness. From this Wesp concludes that overall fiction and video-games should not be separated by the ability of exhibiting incoherence; incoherence is incompleteness and incompleteness is a feature of all forms of fiction.

But it would misconstrue the Mario-reappearance to hold that it is a case of incompleteness in the fictional world. Though we agree with Wesp (as we discuss in the next section) that form-fiction incoherence is not unique to video-games, still we disagree that there is nothing unique about form-fiction incoherence compared to incompleteness.

Let us examine again what happens in the Mario example. Wesp would hold that what is essential about it is that is that we are prevented from gaining a complete understanding of the fictional world. However, we do have a complete understanding of the

4 Interesting in this regard is Vella (2015), where the incompleteness of Dark Souls (From Software 2011) is interpreted as being integral to the game. This is also interesting in general. Incompleteness does not only not have to be a problem; it can actually be used to create good games. Another example would be the choice in horror games to make sure that the player cannot ascertain the exact characteristics of a monster that is lurking in the dark. The incompleteness of the player's knowledge adds to the suspense without detracting from the fiction. 
fictional world. This is exactly because we do not need to explain why Mario dis- and re-appears. We say that Mario, in one go, saved his girlfriend from the gorilla (Juul 2005, 124). And he has done so without disappearing, nor reappearing, and thus also, without reincarnating.

But if Mario did in fact not reincarnate, it cannot be the case that the Mario example is an example of incompleteness; were it to have been a case of incompleteness of the fictional world, we would be required to claim that we do not know whether Mario reincarnated at all. But in the Mario example, what we notice is our explicit disavowal of Mario’s reincarnation.

However, as with contradictions in the game environment, incompleteness of the game environment can be related in specific ways to form-fiction incoherence. In the Mario example we are given proof of reincarnation in the game environment, but through recourse to the rules of the game, we disregard this information. Similarly, when we are not given any information about something by the game environment, the rules of the game may induce us to fill in these gaps in the fictional world. When we find a key in an adventure game, we assume of the fiction of the game that the key will be useful in our adventure (and we do this because we understand that the rules of the game point in this direction, "why else do we find this key in our inventory?”).

So incompleteness of the game environment can be disregarded in favor of a filling in of the fictional world on the basis of the rules of the game. But despite this, as we have argued, incompleteness is not the same as form-fiction incoherence.

\section{Form-fiction Incoherence in Other Media}

Wesp's second claim about what we have called form-fiction incoherence was that other media may just as well exhibit it as do video games. ${ }^{6}$ To argue for this, he gives an example of a television series that is also incoherent in its relation between form and fiction:

Mystery and crime shows, for example, often fail to explain the alarming trail of murder and mayhem that follows their central characters. Without narratively motivated explanation, for instance, the CBS series Murder, She Wrote, depicted the otherwise quaint town of Cabot Cove, Maine as beset by a catastrophic murder rate of which no one takes apparent notice. ( ... ) The logic by which these episodes relate to each other is, in other words, beyond any rational explanation. (Wesp 2014)

5 Juul also discusses examples of this, when we encounter witches in a game for example, we assume them to have magical powers because they are witches in a video game, and not on the basis of what we already knew of witches nor that the game environment has given us proof of the witches having magical powers (Juul 2005, 123).

6 Juul also makes a remark that points in this direction (Juul 2005, 200). 
Here Wesp correctly identifies an occurrence of form-fiction incoherence in another medium. There is striking similarity with the Mario example. Instead of asking "why does Mario have three lives?”, we may ask, "why are there so many murders in Cabot Cove?". Our answer to this question will most probably be along the lines of: "because we are watching a crime series with episodes that need to be interchangeable." ${ }^{7}$ So, Cabot Cove is not really "beset by a catastrophic murder rate," just like Mario does not really have magical powers to whisk him back to life. In the same way, more and more examples can be generated, for many media. Why for example do all the characters in the Iliad speak in rhyme? When we imagine the battlefield around Troy we do not imagine all characters to actually always speak in rhyme. Here we understand that the reason for this is that we are reading an epic poem. And why do some characters in a play appear to be lookalikes? Probably not because we should incorporate into the fiction that king Lear and the Fool are long lost twins, but because both characters are coincidentally played by the same actor.

This shows that other media also exhibit form-fiction incoherence and that it is medium-specific what kind of oddities may be 'forgiven'. In Donkey Kong, we can call upon the rules of the game and that the game would be too difficult otherwise. When watching a series like Murder, She Wrote, we understand that there may be a certain disconnect between the episodes on display, due to it becoming very difficult to watch the different episodes in a random order otherwise. And when reading the Iliad, we accept that some of the words of the characters are to be attributed to that we are reading an epic poem, and not to their mastery of on the spot rhyming. We are in agreement with Wesp's assessment that other media may also exhibit the sort of incoherence we find in the Mario example; however in contrast to Wesp we argued that incoherence should not be understood as incompleteness.

\section{Taking Stock and Redefining Form-fiction Incoherence}

We have noted that incompleteness and contradictions are not identical with formfiction incoherence, precisely because they can be nothing more than a precursor to this phenomenon. However, there is a larger point to be made about form-fiction incoherence versus incompleteness and contradictions. When we say that some game is contradictory or incomplete, we say something about the fictional or game environment of a game. However, when we say that a game is incoherent it is not clear that we ascribe something to the fictional world or the game environment.

In Murder, She Wrote, we might agree with fan-theories that we should incorporate the murder rate in Cabot Cove into the fiction and assert for example that Jessica Fletcher commits the murders herself. However, it seems sensible to hold that the high murder rate is not the case of the fiction exactly because we can understand this to be a product of a long running-time and the requirement for the possible interchange of episodes. 
What occurs in the Mario example? The player is provided with information by the game environment. This information is then interpreted by the player, and most of the information finds its way into the player's understanding of the fiction of the game; the fictional world. However, some events occurring in the game environment are cast aside by the player, they do not find their way into the fictional world. In the Mario example, we disregard that in the game environment Mario dis- and re- appears. In both Juul's and Wesp's account we find attempts at understanding the form-fiction incoherence phenomenon by analysis of the fictional world (it either containing contradictions or incompletions). However, such accounts necessarily leave gaps; since form-fiction incoherence results in a disregarding of information for the fictional world. An analysis of the fictional world cannot suffice, as the interesting nature of the phenomenon is that something does not find its way into the fictional world. We need to incorporate in our account both the specific nature of these disregarded events and the specific sort of player interpretation that underlies it.

In an occurrence of form-fiction incoherence we keep information given to us out of the fictional world. We keep this out of the fictional world on two accounts. Firstly, we understand that the information given in the game environment may be understood as being a part of the rules of the game. Secondly, we understand that there is no palatable interpretation of these events in the game environment possible in the fiction. This means that a full understanding of form-fiction incoherence depends on developing a deeper understanding of the interpretation of the event in the game environment as not part of the fiction. Form-fiction incoherence is not a property of the fictional world, it is a description of a specific interpretative act done by the player which aims at alleviating the tension between the game environment and the fictional world.

We propose to explain form-fiction incoherence as the interpretation of an event as a pseudo fictional event. Based on the previous findings we propose the following understanding of a pseudo fictional event:

A pseudo fictional event is an event presented by the medium that is interpreted by the consumer of the medium to not have occurred in the fictional world. This interpretative act occurs when the consumer of the medium understands that the event can better be attributed to another aspect of the medium.

The concept of "pseudo fictional event", in contrast to incompleteness and contradictoriness of the fictional world, does describe the incoherence found in the Mario example correctly. The player is confronted with Mario's dis- and re- appearing. However, the player understands that this event can be attributed to the rules of the game. The game becomes a better game by having these rules. Thus, the dis- and re-appearing of Mario is not incorporated into the fictional world of Mario. This understanding differs from the incompleteness and contradiction readings we have discussed, because 
these readings of the events are both examples of events that might (when they adhere to the boundary conditions we discussed) be interpreted to be pseudo fictional.

Other media can exhibit pseudo fictional events as well. The Iliad example is a case in point. We understand that the Iliad would be less beautiful were it not in rhyme, and we see that actually attributing to every character who speaks in the Iliad the ability to speak on rhyme would be detrimental to the fictional world of the Iliad. So, we attribute almost all occurrences of first person rhyme to another aspect of the medium, namely being an epic poem.

\section{What's Behind Pseudo Fictional Events: The Principle of Charity}

Although we have now identified that the user need to perform a special kind of interpretation before we can speak of a pseudo fictional event, and that it is through the understanding of this specific way of interpreting that we may expect to gain insight into pseudo fictionality, we have not yet said anything about what kind of principles might underlie this way of interpreting. In his article "On the Very idea of a Conceptual Scheme” (Davidson 1973) the philosopher Donald Davidson introduces such a principle for understanding other people's speech acts. An example:

\footnotetext{
If you see a ketch sailing by and your companion says, "Look at that handsome yawl," you may be faced with a problem of interpretation. One natural possibility is that your friend has mistaken a ketch for a yawl, and has formed a false belief. But if his vision is good and his line of sight favorable it is even more plausible that he does not use the word "yawl" quite as you do, and has made no mistake at all about the position of the jigger on the passing yacht. We do this sort of off the cuff interpretation all the time, deciding in favor or reinterpretation of words in order to preserve a reasonable theory of belief. (Davidson 1973, p.295-296)
}

The principle of charity is introduced by Davidson to solve the problem of radical interpretation. To understand a person's words, we need to know about the person's beliefs, and to know about someone's beliefs, we need to understand his words (Malpas 2015). If we do not assume anything about our companion's beliefs in the example, we would not be able to understand what he means by yawl. Davidson says that we apply the principle of charity in these cases, which states that we should assume our conversational partner to be "as intelligible as possible" (Davidson 2001, xix). In fact there are other assumptions we will also make, for example that the conversational partner is communicating with us. By assuming of both someone's words and beliefs that they are from a person who is intelligible, we can start to make sense of one another's words.

It is common to use this principle of interpretation. When we encounter a monitor filled with train timetables we assume that the creator of what's on the monitor wants to create a timetable that is as good as possible. If the timetable has no entry about a certain train riding between two stations at a certain time, then the traveler will assume that there is in fact no such train at that time. By assuming certain intentions and beliefs of 
the creator, we are capable of deciding that we should assert that there is no train scheduled at that time. ${ }^{8}$

The principle of charity originally required a person to assume that a conversational partner is as intelligible as possible. For our purposes here we will expand on this principle. We can apply the principle of charity to the creator of an artwork in some medium. If we assume that a person who made a game is rational, we can, we claim, also assume that this person did not intend to make a terrible game. ${ }^{9}$ This assumption can then help us with interpreting the medium and can lead to the interpretation of an event as a pseudo fictional one. Let us be clear that this is not a classical application of the principle of charity; the object here is a game (or book, etc.) instead of utterances. This means that we now try to make the piece of media we are presented with as intelligible as possible, which cannot be taken to mean to attribute as many true beliefs as possible anymore. However, the general idea remains; we try to understand the medium we are presented with as charitable as possible.

In the Mario example, we also apply this (deviant) principle of charity. When an event occurs in the game environment that the player understands as being harmful to the cogency, beauty, etc. of the fiction of the game, we can understand that it actually is not part of the fiction, as long as we are capable of seeing its merits for the video game as a game. ${ }^{10}$

In general, when we interact with a game, TV-show etc. we try to understand what we see and hear as being made by a person who wanted to make something good. This means that we interpret the medium with some basic ideas about the creator in mind. When something occurs in the game that reflects poorly on the fiction, but well on the game as rule system, we apply the principle of charity. We assume that since the creator would not have wanted to create poor fiction, this must not be a part of the fiction, but a decision pertaining to the game-mechanics. This way, the occurrence of pseudo fictional

8 This principle that the train traveler is using, is also used by computer scientists who design databases (Raymond 1978). They use the 'Closed World Assumption' when working with and on databases, which states that when something is true, it is known to be true and that when it is not known to be true, it is false. This closely resembles the traveler. He assumes that that which is depicted on the boards is created by someone adhering to this principle. Based on this he can conclude that when there is no information about a train on the board, that there is in fact no such train.

9 Another way to make sense of this would be through Grice's conversational maxims. Although we are not strictly conversing with the creator of the medium, we can understand the piece of media as an attempt of the creator to bring across something. For example, Grice's maxim of relation could explain our expectation of the medium to be to the point and as such, we might expect of certain events in the game environment that they are (at least) either part of the fiction or of the form (Grice 1975).

10 Note that this can occur, whether or not the player is interested in the fiction or not. The pseudo fictionality of events does not depend on a preference of the fiction over the rules of a game. 
events can be understood as an application of the principle of charity on a piece of media.

\section{Pseudo Fictionality as a Design Tool}

Do we want our games to trigger the interpretation of some events as pseudo fictional events? At first sight the answer seems to be no. Wesp puts it like this:

First, the pronouncement of a fiction as "incoherent" short-circuits the possibility of its meaning, seeking only to explain why the fiction fails. (Wesp 2014)

However the possibility for finding the game meaningful is not put in peril by the interpretation of Mario's seeming reincarnation as a pseudo fictional event. It is the other way around; due to the interpretation of the reincarnation as pseudo fictional, we save the game's fiction. For, after having taken reincarnation as being pseudo fictional, we can now tell a sensible story about what has happened to Mario. Mario's fictional world is saved from the un-elegant story element of reincarnation. Something which would have taken away all tension when we were to retell the story to others is no longer true of the fictional world. "Mario had to overcome perilous obstacles to save his girlfriend, while in constant danger of dying, having no second chances to rely on" is the preferable fiction we are left with when we understand the reincarnation as pseudo fictional. So in the end the fiction does not fail, precisely because the event is understood as being pseudo fictional. And Juul asserts this as well, pointing to that understanding an event as pseudo fictional (incoherent) does not break down our understanding of the fictional world at all, it simply foregrounds the rules (or some media's equivalent):

Still, if we focus on the rules of the game, the experience of playing Donkey Kong is not incoherent. (Juul 2005, 130)

And in the chapter "What a Game Means":

Breaking the coherence of the fictional world does not so much foreground the way the game projects a fictional world as it foregrounds the rules, the game as an activity. In a multiplayer game, breaking the coherence of the fictional world can work as a foregrounding of the game as a real-life social activity. (Juul 2005, 195)

When we look at pseudo fictional events from the creator's perspective, they can be conceived of as design tools. A creator commonly includes both fiction and rules in the game, which can be challenging to combine. The idea behind using knowledge of pseudo fictional events in the design of games is to purposefully trigger them. By purposefully triggering the interpretation of an event in the game environment as pseudo fictional a creator can include rules in the game that produce events in the game environment which when taken to be true of the fictional world would be detrimental for the fiction. Taking this event as pseudo fictional helps the creator precisely because it is barred from the fictional world. The pseudo fictional status of the events caused by the game's rules ensures that the player does not take these rules as part of the fiction. There 
are various reasons why creators want to include rules which do not align with the fiction. For example, the respawning in Donkey Kong may be seen as a way to reduce the frustration of dying, but at the same time, the designer does not want to introduce the tension-reducing element of Mario's capacity for revival into the narrative. There may be other interesting uses such as the foregrounding of rules to create humor. ${ }^{11}$

In some cases the player may not do the pseudo fictional interpretative act, against the intention of the creator. In this case, the rules are strange in the context of the fictional world. We see strong similarities between this scenario and the much debated ludonarrative dissonance (Hocking 2009). Ludonarrative dissonance describes a problematic conflict between the game rules and the narrative. It occurs for example when a main character is portrayed as a likable sane person in the narrative, while the game rules require the main character to kill of hundreds of other characters in often brutal ways. But while ludonarrative dissonance is described as a property of a game, our examination of pseudo fictional events suggests that the specifics of the player's interpretation are essential. We hypothesize that a focus on the player may explain the amount of disagreement on ludonarrative dissonance: some players do experience it, while others do not. This explains why some players do not experience the dissonance: they simply do not attribute the conflicting rules to the fictional world.

\section{Conclusion}

In this paper we have argued that incompleteness and contradiction do not give rise to sensible ways of understanding of form-fiction incoherence. This was argued by showing how these interpretations do not allow for a correct description of the paradigmatic Mario example. Because of this we proposed to redefine form-fiction incoherence as the occurrence of a pseudo fictional event. This way of understanding the phenomenon of form-fiction incoherence does neatly describe the Mario example, as well as a number of other occurrences of form-fiction incoherence in other media (Murder She Wrote, The Iliad, King Lear). The notion of a pseudo fictional event brought forth the importance of the interpretative act done by the player. This in turn allowed us to investigate what sort of interpretations might underlie the occurrence of pseudo fictional events. To understand these interpretations we have discussed the principle of charity as applied to fiction: we try to interpret media (both its form and its fiction) in as positive "charitable" way as possible. Finally, we have discussed the potential of the notion of pseudo fictionality to understand the design of games and ludonarrative dissonance.

11 In the analysis of humor in adventure games, Giappone (2015) discovers methods that arguably make use of pseudo fictionality. To produce humor, the game at times pulls the focus of the player towards the rules of the game. To do this, something odd is made to happen, so that the player is pulled out of the fiction in search of an explanation in the game rules. And in the game's rules some meta-joke is situated. 


\section{Media}

From Software. 2011. Dark Souls. [Playstation 3]. Namco Bandai Games.

Nintendo. 1981. Donkey Kong [Arcade]. Nintendo.

Murder, She Wrote. Directed by Angela Lansbury, Peter Fischer, Richard Levinson, William Link and David Moessinger. CBS, 1984-1996.

\section{References}

Davidson, Donald. 1973. “On the very idea of a Conceptual Scheme.” In The Pragmatism Reader, edited by Robert Talisse and Scott Aikin, 286-298. Princeton, Woodstock: Princeton University Press.

- 2001. Inquiries into Truth and Interpretation. 2nd ed. Oxford: Clarendon Press.

Giappone, Krista. 2015. "Self-Reflexivity and Humor in Adventure Games." Game Studies 15, no. 1. http://gamestudies.org/1501.

Grice, Paul. 1975. "Logic and Conversation.” In Speech Acts, edited by P. Cole and J. Morgan, 4158. New York: Academic Press.

Hocking, Clint. 2009. "Ludonarrative Dissonance in Bioshock: The Problem of What the Game is About.” In Well Played 1.0, edited by Draw Davidson, 255-260. Pittsburgh, PA, USA: ETC Press.

Homer. 1998. The Iliad, trans. Robert Fagles. New York: Penguin Books.

Juul, Jesper. 2005 Half-Real. Cambridge, London: MIT Press.

Malpas, Jeff. 2015. "Donald Davidson", The Stanford Encyclopedia of Philosophy (Fall 2015 Edition), edited by Edward N. Zalta. http://plato.stanford.edu/archives/fall2015/entries/davidson/.

Pavel, Thomas. 1986. Fictional Worlds. Cambridge, London: President and Fellows of Harvard College.

Polansky, Lana. 2015 “Coherence and Dissonance.” Sufficiently Human. http://sufficientlyhuman.com/archives/1006.

Reiter, Raymond. 1978. "On Closed World Data Bases". In Logic and Data Bases, edited by Hervé Gallaire and Jack Minker, 119-140. Plenum Press.

Shakespeare, William. 1994. King Lear. Herfordshire: Wordsworth Editions Limited.

Thon, Jan-Noël. 2009. "Computer Games, Fictional Worlds, and Transmedia Storytelling: A Narratological perspective”, In Proceedings of the Philosophy of Computer Games Conference, Oslo.

Vella, Daniel. 2015. "No Mastery Without Mystery: Dark Souls and the Ludic Sublime”, Game Studies 15, no. 1. http://gamestudies.org/1501.

Wesp, Edward. 2014. "A Too-Coherent World: Game Studies and the Myth of "Narrative” Media." Game Studies 14, no. 2. http://gamestudies.org/1402. 